\title{
Ultrahigh-energy cosmic rays particle spectra from crypton decays
}

\author{
John Ellis, ${ }^{1}$ V. E. Mayes, ${ }^{2}$ and D. V. Nanopoulos ${ }^{2,3}$ \\ ${ }^{1}$ Theory Division, Physics Department, CERN, CH 1211 Geneva 23, Switzerland \\ ${ }^{2}$ George P. and Cynthia W. Mitchell Institute for Fundamental Physics, Texas A\&M University, College Station, Texas 77843, USA \\ ${ }^{3}$ Astroparticle Physics Group, Houston Advanced Research Center (HARC), Mitchell Campus, Woodlands, Texas 77381, USA \\ and Academy of Athens, Division of Natural Sciences, 28 Panepistimiou Avenue, Athens 10679, Greece
}

(Received 13 February 2006; published 7 December 2006)

\begin{abstract}
We calculate the spectra of ultrahigh-energy cosmic rays (UHECRs) in an explicit top-down model based on the decays of metastable neutral "crypton" states in a flipped SU(5) string model. For each of the eight specific 10th-order superpotential operators that might dominate crypton decays, we calculate the spectra of both protons and photons, using a code incorporating supersymmetric evolution of the injected spectra. For all the decay operators, the total UHECR spectra are compatible with the available data. Also, the fractions of photons are compatible with all the published upper limits, but may be detectable in future experiments.
\end{abstract}

DOI: 10.1103/PhysRevD.74.115003

\section{INTRODUCTION}

The existence of cosmic rays with energies above the Greisen-Zatsepin-Kuzmin (GZK) cutoff [1,2] is one of the most important open problems in high-energy astrophysics $[3,4]$. These ultrahigh-energy cosmic rays (UHECRs) may be a tantalizing hint of novel and very powerful astrophysical accelerators, or they may be harbingers of new microphysics via the decays of metastable supermassive particles. It is remarkable that we still do not know whether the UHECRs originate from macrophysics or microphysics. If there is no GZK cutoff, as suggested by the AGASA data [5], the sources of the UHECRs would need to be local. In this case, since local magnetic fields are unlikely to have deflected significantly their directions of propagation, the UHECRs would "remember" the directions of their sources. Thus, one would expect some anisotropy in the arrival directions of the UHECRs, associated either with discrete energetic astrophysical sources nearby, such as BL Lac objects [4], or the distribution of (mainly galactic) superheavy dark matter. No significant anisotropy of the UHECRs has yet been seen, but the existing experiments have insufficient statistics to exclude one at the expected level [6]. On the other hand, the GZK cutoff may be present in the HiRes data [7], in which case no exotic microphysics may be needed, and any astrophysical sources would be less restricted and more difficult to trace. The first batch of Auger [8] data are inconclusive on the possible existence of the GZK cutoff.

Superheavy particles of the type required could have been produced gravitationally around the end of inflation [9]. Particularly interesting candidates for such superheavy particles are "cryptons," bound states of the fractionally charged constituents that arise generically [10] in the hidden sectors of models of particle physics derived from the heterotic string. ${ }^{1}$ Cryptons arising in the hidden sector of a

\footnotetext{
${ }^{1}$ Such states may also be a generic feature of models constructed from intersecting $D$-branes.
}

PACS numbers: 12.60.Jv, 04.60. $-\mathrm{m}, 11.25 . \mathrm{Wx}, 96.50 . \mathrm{S}-$

heterotic string-derived flipped SU(5) model may have exactly the right properties to play this role $[11,12]$. These "flipped cryptons" are bound by SU(4) hiddensector interactions, and include four-constituent metastable bound states called tetrons that are analogues of the threeconstituent baryons of QCD, as well as two-constituent mesonlike states. Indeed, it was within this flipped SU(5) model that the confinement solution to avoiding the stringent experimental limits placed on fractional charges was first pointed out, and this model remains the only example to have been worked through in any detail $[11,12]$.

In general, tetrons may decay through $N$ th-order nonrenormalizable operators in the superpotential, which would yield lifetimes that are expected to be of the order of

$$
\tau \approx \frac{\alpha_{\mathrm{string}}^{2-N}}{m_{X}}\left(\frac{M_{s}}{m_{X}}\right)^{2(N-3)}
$$

where $m_{X}$ is the tetron mass and $M_{S} \sim 10^{18} \mathrm{GeV}$ is the string scale. The $\alpha$-dependent factor reflects the expected dependence of high-order superpotential terms on the effective gauge coupling $g$. The mass scale associated with these states is estimated using the renormalization group for the SU(4) interactions to be $\Lambda \sim 10^{12}-10^{13} \mathrm{GeV}$, just in the right range for their decays to produce the UHECRs.

The lifetimes of neutral tetrons without electric charge has been estimated to lie in the range $\tau_{0} \sim 10^{11}-10^{17}$ years, so that they may still be present in the Universe today, and might produce the necessary flux of UHECRs if they are sufficiently abundant. We have shown in our previous work [12] that the mesons and charged tetrons - whose presentday abundances are subject to very stringent experimental limits - would have decayed with short lifetimes early in the Universe. In the course of studying the possible tetron lifetimes, we identified various 10th-order superpotential operators that might govern neutral tetron decays. Thus, in this specific model of flipped cryptons we are able to go beyond generic statements regarding the injected UHECR 
particle spectra that may result from their decays, and make a number of specific predictions.

This enables us to address an important experimental constraint on such crypton models of the UHECRs. Although "top-down" models such as crypton decays may appear to be natural explanations for the UHECRs (if they exist), they generically share a potential drawback. The spectra of UHECRs that they produce might be expected to have large photon fractions, in possible conflict with the observation that most of the UHECR primaries appear to be protons or nuclei. The Auger collaboration has recently set an upper limit of $26 \%$ on the photon fraction above $10^{19} \mathrm{eV}$ [13], and the limit may even be as low as $7 \%-14 \%$ [14]), while an upper limit of 50\% at energies above $10^{20} \mathrm{GeV}$ has been set by the AGASA collaboration [15].

In this paper, we first give a review of relevant aspects of the flipped SU(5) heterotic string model. We then analyze the specific primary multibody decay modes governed by the various different 10th-order superpotential operators found in our previous paper. We then calculate the UHECR particle spectra that would be injected by these decays using one of the most detailed and complete codes currently available [16], paying particular attention to the photon fraction. The total UHECR spectra obtained from the various superpotential terms do not differ greatly. On the other hand, we find that different decay operators may give rather different photon fractions, particularly at the highest energies. However, in every case, we find that the calculated spectra after supersymmetric evolution and fragmentation are compatible with the published upper limits on the photon fractions in various energy ranges, when we include the UHECR background resulting from a homogenous extragalactic distribution of sources and incorporate the pileup expected from the GZK effect. There is no need to appeal to the cosmic radio background to absorb a significant fraction of the photons in order to bring them below the AGASA and other limits.

\section{GENERIC SUPERHEAVY RELIC DECAY}

The basic idea in generic top-down explanations (see $[17,18]$ ) is that the UHECR are produced via the decay of some relic particles or topological defects left over from the inflationary epoch and which are locally clustered in the galactic halo as cold, dark matter with an overdensity $n_{X} / n_{X}^{\text {cos }} \sim 10^{4-5}$. The lifetime of such relics must exceed the present age of the Universe in order for them to exist today in sufficient abundance, however the lifetime must not be too large so that the decay rates produced are too small to produce the UHECR. Furthermore, the relic mass must be at least $M_{X}>10^{12} \mathrm{GeV}$ in order to produce the UHECR energies observed. Typically, the lifetime of a particle is expected to be inversely proportional to its mass, $\tau \sim 1 / M$. Clearly it is not easy to have a particle with both a large enough mass and a decay lifetime in the right range to produce the UHECR. However, as pointed out in the introduction, flipped SU(5) cryptons satisfy both of these criteria, which makes them very attractive as a topdown explanation of the UHECR. Indeed the flipped crypton is probably the most natural and most physically motivated of any top-down candidate.

There are three generic statements that can be presently made about a decaying superheavy $X$ particle explanation for the UHECR:

(1) Since the superheavy relics may accumulate locally in the galactic halo with an overdensity $\sim 10^{4-5}$ over the cosmological average, they may avoid the GZK cutoff.

(2) Because of the displacement of our solar system with respect to the galactic plane, there should be some anisotropy in the arrival directions of the UHECR with respect to the galactic center.

(3) Photons tend to be the dominant component of the UHECR flux produced by the superheavy $X$ decay. However, the photons may scatter off the galactic radio background, which is poorly measured, and thus may be somewhat attenuated.

The injection spectrum produced by such a decaying superheavy relic $X$ particles with number density $n_{X}$ and lifetime $\tau_{X}$ is proportional to the inclusive decay width:

$$
\Phi^{\text {halo }}(E)=\frac{n_{X}}{\tau_{X}} \frac{1}{\Gamma_{X}} \frac{d \Gamma\left(X \rightarrow g_{1}+\cdots\right)}{d E} .
$$

If a spherical halo of radius $R_{\text {halo }}$ and uniform number density $n_{X}$ is assumed, then the galactic halo contribution to the UHECR will be given by

$$
J^{\text {halo }}=\frac{1}{4 \pi} R_{\text {halo }} \Phi^{\text {halo }}(E) .
$$

In general, the $X$ particles will decay into one or more partons which hadronize into other particles $g$ of the minimal supersymmetric standard model (MSSM), which carry a fraction $x$ of the maximum available momentum $M_{X} / 2$ and a fraction $z$ of the parton momentum. For such a decay, the inclusive decay width can be factored as

$$
\begin{aligned}
\frac{1}{\Gamma_{X}} \frac{d \Gamma\left(X \rightarrow g_{1}+\cdots\right)}{d E}= & \left.\sum_{a} \int_{0}^{x} \frac{1}{\Gamma_{a}} \frac{d \Gamma_{a}\left(y, \mu^{2}, M_{X}^{2}\right)}{d y}\right|_{y=x / z} \\
& \times D_{a}^{g}\left(z, \mu^{2}\right),
\end{aligned}
$$

where $D_{a}^{g}\left(z, \mu^{2}\right)$ is the fragmentation function (FF) for particles of type $g$ into particles of type $a$, and $\mu$ is the energy scale, most appropriately taken to be equal to the $X$ particle mass, $\mu=M_{X}$. The evolution of the fragmentation function is governed by the Dokshitzer-GribovLipatov-Altarelli-Parisi (DGLAP) equations, which may be extended to include the MSSM. Thus, the determination of the expected UHECR flux from the superheavy $X$ decay essentially becomes the problem of starting with a set of initial decay partons and evolving the decay cascades via the fragmentation functions to find the end decay products 
and energy distribution. To evolve the fragmentation functions up to the energy of the superheavy $X$ decay, the DGLAP equations must be solved numerically. Several groups have done such calculations for generic initial decay partons (usually into a quark-antiquark pair) [1922]. Perhaps the best such code is SHdecay [16]. This code calculates the fragmentation into the seven stable MSSM particles $\left(p, \gamma, e\right.$, neutralino LSP $\left.\chi, \nu_{e}, \nu_{\mu}, \nu_{\tau}\right)$ for any given initial decay parton. In the case of flipped SU(5) cryptons, we have a specific model where the initial decay partons in the cascade are known.

In addition to the UHECR flux produced by the superheavy decay from $X$ particles clustered in our galactic halo, there may be a background flux from sources outside of our galaxy, perhaps superheavy $X$ decay in other galactic halos or in intergalactic regions. Generally, this flux is assumed to be due to a homogenous distribution of sources and exhibits a characteristic GZK pileup due to the fact that they are produced nonlocally [23]. Since the GZK attenuation is much more severe for photons than for nucleons, this background should be comprised primarily of nucleons. Thus, in this scenario the UHECR flux observed on Earth will be the sum of this extragalactic background and the local galactic flux from decaying relics clustered in our galactic halo. Because of the extragalactic component, superheavy $X$ particle decay may only unambiguously explain the UHECR flux for energies $E>4 \times 10^{19} \mathrm{eV}$. However, we note that the extragalactic component may also be due partially to nonlocal superheavy $X$ decay, as well from astrophysical sources ("bottom-up" production). Thus, it is more accurate to say that a distinct signal of superheavy $X$ decay within our galactic halo would be the existence of an excess of events above this energy. The lack of any events above this energy would not rule out the presence of a top-down component, but it would not provide an unambiguous reason for the introduction of such a mechanism.

\section{STRING-DERIVED FLIPPED SU(5)}

Even before the first string models, there was strong interest in flipped $\mathrm{SU}(5)$ as a possible grand unified theory (GUT), primarily because it did not require large Higgs representations and avoided the constraints of minimal SU(5) [24] without the extra gauge interactions required in larger groups such as $\mathrm{SO}(10)[25,26]$. Interest in flipped SU(5) increased within the context of string theory, since simple string constructions could not provide the adjoint and larger Higgs representations required by other grand unified theories, whereas they could provide the Higgs representations required for flipped SU(5). It was, moreover, observed that flipped SU(5) provided a natural "missing-partner" mechanism for splitting the electroweak-doublet and color-triplet fields in its fivedimensional Higgs representations [27]. Heterotic string- derived flipped $\mathrm{SU}(5)$ remains among the most fully developed and realistic models yet derived from string.

Flipped SU(5) derives its name from the flipping of the quark and lepton assignments relative to those in the minimal SU(5) GUT [24]: $u_{L}, u_{L}^{c} \leftrightarrow d_{L}, d_{L}^{c}$ and $\nu_{L}, \nu_{L}^{c} \leftrightarrow$ $e_{L}, e_{L}^{c}$ for all three generations. This requires the gauge group to become $\mathrm{SU}(5) \times \mathrm{U}(1)$, so as to accommodate the charges of the quarks and leptons, which fill up the $\overline{\mathbf{5}}, \mathbf{1 0}$, and $\mathbf{1}$ representations of $\mathrm{SU}(5)$ :

$$
\begin{gathered}
f_{\overline{\mathbf{5}}}=\left(\begin{array}{c}
u_{1}^{c} \\
u_{2}^{c} \\
u_{3}^{c} \\
e \\
\nu_{e}
\end{array}\right)_{L} \quad F_{\mathbf{1 0}}=\left(\left(\begin{array}{l}
u \\
d
\end{array}\right)_{L} d_{L}^{c} \quad \nu_{L}^{c}\right) ; \\
l_{\mathbf{1}}^{c}=e_{L}^{c} .
\end{gathered}
$$

The presence of a neutral component in the $\mathbf{1 0}$ allows spontaneous GUT symmetry breaking to be achieved using a $\mathbf{1 0}$ and a $\overline{\mathbf{1 0}}$ of superheavy Higgs fields with the same and opposite $U(1)$ hypercharges. Their neutral components develop large vacuum expectation values (vevs) breaking $\mathrm{SU}(5) \times \mathrm{U}(1) \rightarrow \mathrm{SU}(3) \times \mathrm{SU}(2) \times \mathrm{U}(1)$, while the electroweak spontaneous breaking occurs through the Higgs doublets $\mathbf{H}_{2}$ and $\mathbf{H}_{\overline{2}}$ :

$$
h_{\mathbf{5}}=\left\{\mathbf{H}_{2}, \mathbf{H}_{3}\right\} ; \quad h_{\overline{\mathbf{5}}}=\left\{\mathbf{H}_{\overline{\mathbf{2}}}, \mathbf{H}_{\overline{3}}\right\} .
$$

The resulting economical doublet-triplet splitting mechanism gives a large mass to the Higgs triplets $\left(\mathbf{H}_{3}, \mathbf{H}_{\overline{3}}\right)$ by coupling them to states in $\mathbf{1 0}$ and $\mathbf{1 0}$ Higgs representations, through trilinear superpotential couplings of the form

$$
\begin{aligned}
& F F h \rightarrow d_{H}^{c}\left\langle\nu_{H}^{c}\right\rangle H_{3}, \\
& \bar{F} \bar{F} \bar{h} \rightarrow \bar{d}_{H}^{c}\left\langle\bar{\nu}_{H}^{c}\right\rangle H_{\overline{3}},
\end{aligned}
$$

while keeping light the Higgs doublets $\left(\mathbf{H}_{2}, \mathbf{H}_{\overline{2}}\right)$ light.

The absence of any mixing between the Higgs triplets $\left(\mathbf{H}_{3}, \mathbf{H}_{\overline{3}}\right)$ in this dynamic doublet-triplet splitting mechanism provides a natural suppression of the $d=5$ operators that might otherwise mediate rapid proton decay, so that flipped SU(5) is probably the simplest unified model that can satisfy the experimental limits placed on the proton lifetime [28].

String-derived flipped SU(5) belongs to a class of models constructed using free fermions on the world sheet, corresponding to compactification on the $Z_{2} \times Z_{2}$ orbifold at the maximally symmetric point in the Narain moduli space [29]. Although this model was originally constructed in the weak-coupling limit, it is possible that it may be elevated in the strong-coupling limit to an authentic $M$ theory model and may at some point make contact with models based on $D$-brane constructions.

The full gauge group of the model is $\mathrm{SU}(5) \times \mathrm{U}(1) \times$ $\mathrm{U}(1) 4 \times \mathrm{SO}(10) \times \mathrm{SO}(6)[\simeq \mathrm{SU}(4)]$, where the latter two factors are confining hidden-sector gauge interactions. The matter spectrum comprises the following fields: 
(i) Observable sector: The conventional matter fields may be regarded as three $\mathbf{1 6}$ representations of $\mathrm{SO}(10)$ that $\supset \mathrm{SU}(5) \times \mathrm{U}(1)$ chiral multiplets $F_{i}\left(\mathbf{1 0}, \frac{1}{2}\right), \bar{f}_{i}\left(\overline{\mathbf{5}},-\frac{3}{2}\right), l_{i}^{c}\left(\mathbf{1}, \frac{5}{2}\right)(i=1,2,3)$; extra matter fields $F_{4}\left(\mathbf{1 0}, \frac{1}{2}\right), f_{4}\left(\mathbf{5}, \frac{3}{2}\right), \bar{l}_{4}^{c}\left(\mathbf{1},-\frac{5}{2}\right)$ and $\bar{F}_{5}\left(\overline{\mathbf{1 0}},-\frac{1}{2}\right)$, $\bar{f}_{5}\left(\overline{\mathbf{5}},-\frac{3}{2}\right), l_{5}^{c}\left(\mathbf{1}, \frac{5}{2}\right)$; and four Higgs-like fields in the 10 representation of $\mathrm{SO}(10)$, that $\supset h_{i}(\mathbf{5},-1)$, $\bar{h}_{i}(\overline{\mathbf{5}}, 1), i=1,2,3,45$. In our realization of the model, we make the following flavor identifications of the standard model states with the various string representations:

$$
\begin{gathered}
t b \tau \nu_{\tau}: Q_{4} d_{4}^{c} u_{5}^{c} L_{1} l_{1}^{c}, \quad \operatorname{cs} \mu \nu_{\mu}: Q_{2} d_{2}^{c} u_{2}^{c} L_{2} l_{2}^{c}, \\
u d e \nu_{e}: Q_{\beta} d_{\beta}^{c} u_{1}^{c} L_{5} l_{5}^{c},
\end{gathered}
$$

where $\beta$ indicates a mixture of fields with the indices 1 and 3. The light Higgs doublets $h_{d}$ and $h_{u}$ (where $h_{d}$ is the Higgs doublet responsible for giving mass to the down-type quarks and leptons and $h_{u}$ gives mass to the up-type quarks) are contained in the $h_{1}$ and $\bar{h}_{45}$ string pentaplet representations, respectively.

(ii) Singlets: There are ten gauge-singlet fields $\phi_{45}, \phi^{+}$, $\phi^{-}, \phi_{i}(i=1,2,3,4), \Phi_{12}, \Phi_{23}, \Phi_{31}$, their ten "barred" counterparts, and five extra fields $\Phi_{I}(I=$ $1 \cdots 5)$.

(iii) Hidden sector: This contains 22 matter fields in the following representations of $S O(10)_{h} \otimes S U(4)_{h}$ : $T_{i}(\mathbf{1 0}, \mathbf{1}), \Delta_{i}(\mathbf{1}, \mathbf{6})(i=1 \cdots 5) ; \tilde{F}_{i}(\mathbf{1}, \mathbf{4}), \tilde{\bar{F}}_{i}(\mathbf{1}, \overline{\mathbf{4}}) \times$ $(i=1 \cdots 6)$. Flat potential directions along which the anomalous combination of hypercharges $\mathrm{U}(1)_{A}$ are cancelled induce masses that are generally near the string scale for some, but not all, of these states. Depending upon the number of $T_{i}$ and $\Delta_{i}$ states remaining massless, the $\mathrm{SO}(10)$ condensate scale is $10^{14-15} \mathrm{GeV}$ and the SU(4) condensate scale is $10^{11-13} \mathrm{GeV}$ [30]. The $\tilde{F}_{3,5}$ and $\tilde{\bar{F}}_{3,5}$ states always remain massless down to the condensate scale. The $\mathrm{U}(1)_{i}$ charges and hypercharge assignments are shown in the Table I below.

The hidden-sctor matter fields are confined into crypton bound states. These occur in "cryptospin" multiplets with different permutations of confined constituents, analogous to the flavor SU(2), SU(3), and SU(4) multiplets of bound states in QCD. The cryptospin multiplets contain doubly charged tetrons

$$
\begin{array}{ll}
\Psi^{--}=\tilde{F}_{3} \tilde{F}_{3} \tilde{F}_{3} \tilde{F}_{3}, & \Psi^{++}=\tilde{F}_{5} \tilde{F}_{5} \tilde{F}_{5} \tilde{F}_{5}, \\
\bar{\Psi}^{++}=\tilde{\bar{F}}_{3} \tilde{\bar{F}}_{3} \tilde{\bar{F}}_{3} \tilde{\bar{F}}_{3}, & \bar{\Psi}^{--}=\tilde{\bar{F}}_{5} \tilde{\bar{F}}_{5} \tilde{\bar{F}}_{5} \tilde{\bar{F}}_{5},
\end{array}
$$

TABLE I. The spectrum of hidden matter fields that are massless at the string scale in the revamped flipped SU(5) model. We display the quantum numbers under the hidden gauge group $\mathrm{SO}(10) \times \mathrm{SO}(6) \times \mathrm{U}(1)^{4}$, and subscripts indicate the electric charges.

\begin{tabular}{ll}
\hline \hline$\Delta_{1}^{0}\left(0,1,6,0,-\frac{1}{2}, \frac{1}{2}, 0\right)$ & $\Delta_{2}^{0}\left(0,1,6,-\frac{1}{2}, 0, \frac{1}{2}, 0\right)$ \\
$\Delta_{3}^{0}\left(0,1,6,-\frac{1}{2},-\frac{1}{2}, 0, \frac{1}{2}\right)$ & $\Delta_{4}^{0}\left(0,1,6,0,-\frac{1}{2}, \frac{1}{2}, 0\right)$ \\
$\Delta_{5}^{0}\left(0,1,6, \frac{1}{2}, 0,-\frac{1}{2}, 0\right)$ & \\
$\Delta_{1}^{0}\left(10,1,0,-\frac{1}{2}, \frac{1}{2}, 0\right)$ & $\Delta_{2}^{0}\left(10,1,-\frac{1}{2}, 0, \frac{1}{2}, 0\right)$ \\
$\Delta_{3}^{0}\left(10,1,-\frac{1}{2},-\frac{1}{2}, 0, \frac{1}{2}\right)$ & $\Delta_{4}^{0}\left(10,1,0, \frac{1}{2},-\frac{1}{2}, 0\right)$ \\
$\Delta_{5}^{0}\left(10,1,-\frac{1}{2}, 0, \frac{1}{2}, 0\right)$ & \\
\hline \hline \hline$\tilde{F}_{1}^{+(1 / 2)}\left(1,4,-\frac{1}{4}, \frac{1}{4},-\frac{1}{4}, \frac{1}{2}\right)$ & $\tilde{F}_{2}^{+(1 / 2)}\left(1,4,-\frac{1}{4}, \frac{1}{4},-\frac{1}{4},-\frac{1}{2}\right)$ \\
$\tilde{F}_{3}^{-(1 / 2)}\left(1,4, \frac{1}{4}, \frac{1}{4},-\frac{1}{4}, \frac{1}{2}\right)$ & $\tilde{F}_{4}^{+(1 / 2)}\left(1,4, \frac{1}{4},-\frac{1}{4},-\frac{1}{4}, 6-\frac{1}{2}\right)$ \\
$\tilde{F}_{5}^{+(1 / 2)}\left(1,4,-\frac{1}{4}, \frac{3}{4},-\frac{1}{4}, 0\right)$ & $\tilde{F}_{6}^{+(1 / 2)}\left(1,4,-\frac{1}{4}, \frac{1}{4},-\frac{1}{4},-\frac{1}{2}\right)$ \\
$\tilde{\bar{F}}_{1}^{-(1 / 2)}\left(1,4,-\frac{1}{4}, \frac{1}{4}, \frac{1}{4}, \frac{1}{2}\right)$ & $\tilde{\bar{F}}_{2}^{-(1 / 2)}\left(1,4,-\frac{1}{4}, \frac{1}{4}, \frac{1}{4},-\frac{1}{2}\right)$ \\
$\tilde{\bar{F}}_{3}^{+(1 / 2)}\left(1,4,-\frac{1}{4},-\frac{1}{4}, \frac{1}{4},-\frac{1}{2}\right)$ & $\tilde{\bar{F}}_{3}^{-(1 / 2)}\left(1,4,-\frac{1}{4}, \frac{1}{4}, \frac{1}{4},-\frac{1}{2}\right)$ \\
$\tilde{\bar{F}}_{5}^{-(1 / 2)}\left(1,4,-\frac{3}{4}, \frac{1}{4},-\frac{1}{4}, 0\right)$ & $\tilde{\tilde{F}}_{6}^{-(1 / 2)}\left(1,4, \frac{1}{4},-\frac{1}{4}, \frac{1}{4},-\frac{1}{2}\right)$ \\
\hline
\end{tabular}

and singly charged tetrons

$$
\begin{array}{ll}
\Psi^{+}=\tilde{F}_{3} \tilde{F}_{5} \tilde{F}_{5} \tilde{F}_{5}, & \Psi^{-}=\tilde{F}_{5} \tilde{F}_{3} \tilde{F}_{3} \tilde{F}_{3}, \\
\bar{\Psi}^{-}=\tilde{\bar{F}}_{3} \tilde{\bar{F}}_{5} \tilde{\bar{F}}_{5} \tilde{\bar{F}}_{5}, & \bar{\Psi}^{+}=\tilde{\bar{F}}_{5} \tilde{\bar{F}}_{3} \tilde{\bar{F}}_{3} \tilde{\bar{F}}_{3},
\end{array}
$$

as well as neutral tetrons

$$
\Psi^{0}=\tilde{F}_{3} \tilde{F}_{3} \tilde{F}_{5} \tilde{F}_{5}, \quad \bar{\Psi}^{0}=\tilde{\bar{F}}_{3} \tilde{\bar{F}}_{3} \tilde{\bar{F}}_{5} \tilde{\bar{F}}_{5} .
$$

We have shown previously that the charged tetrons could have decayed with rather short lifetimes early in the history of the Universe [12], and so avoid any problems with charged dark matter particles [31].

However, the neutral tetrons can decay only via higherorder operators in the superpotential, which we have identified in [12], and may have lifetimes exceeding the age of the Universe. This may make them good candidates for cold dark matter, and their slow decays are a possible source of the UHECRs.

In the next section, we turn our attention to these interactions and examine the UHECR energy spectra that may result from these specific tetron decays.

\section{UHECR INJECTION SPECTRA}

We have previously found [12] the following 10th-order superpotential operators through which the neutral tetrons may decay:

$$
\begin{aligned}
& \Psi^{0}\left[F_{2} F_{2} \bar{\Phi}_{31} \bar{\phi}_{45} \phi^{-} h_{1}+F_{2} F_{2} \Phi_{23} \bar{\phi}_{45} \bar{\phi}^{+} h_{1}+F_{2} F_{3} F_{3} \phi_{4} \bar{\phi}_{45} \bar{f}_{2}+F_{4} \Phi_{23} \bar{\phi}_{45} \phi^{-} \bar{h}_{45} \bar{f}_{5}+\left(\bar{\Phi}_{31} \bar{\phi}_{45} \phi^{-}\right.\right. \\
& \left.\left.\quad+\Phi_{23} \bar{\phi}_{45} \bar{\phi}^{+}\right) h_{1}\left(\bar{f}_{2} l_{2}^{c}+\bar{f}_{5} l_{5}^{c}\right)+\Phi_{23} \bar{\phi}_{45} \phi^{-} h_{1} \bar{f}_{1} l_{1}^{c}\right],
\end{aligned}
$$




$$
\begin{aligned}
\bar{\Psi}^{0} & {\left[F_{2} F_{2} \Phi_{31} \phi_{45} \bar{\phi}^{-} h_{1}+F_{2} F_{2} \bar{\Phi}_{23} \phi_{45} \phi^{+} h_{1}+F_{2} F_{2} \bar{\phi}^{-} h_{1} h_{1} \bar{h}_{45}+F_{4} F_{4} \Phi_{31} \phi_{45} \phi^{+} h_{1}+F_{4} F_{4} \phi^{+} h_{1} h_{1} \bar{h}_{45}\right.} \\
& +F_{4} \Phi_{31} \phi_{45} \phi^{+} \bar{h}_{45} \bar{f}_{5}+F_{4} \phi^{+} h_{1} \bar{h}_{45} \bar{h}_{45} \bar{f}_{5}+F_{4} \bar{\phi}^{-} h_{1} h_{1} h_{1} l_{5}^{c}+\left(\Phi_{31} \phi_{45} \phi^{+} h_{1}+\phi^{+} h_{1} h_{1} \bar{h}_{45}\right) \bar{f}_{1} l_{1}^{c} \\
& \left.+\left(\Phi_{31} \phi_{45} \bar{\phi}^{-} h_{1}+\bar{\Phi}_{23} \phi_{45} \phi^{+} h_{1}+\phi^{+} \bar{h}_{45} \bar{h}_{45} \bar{h}_{45}+\bar{\phi}^{-} h_{1} h_{1} \bar{h}_{45}\right)\left(\bar{f}_{2} l_{2}^{c}+\bar{f}_{5} l_{5}^{c}\right)\right] .
\end{aligned}
$$

Using the flavor identifications we outlined above, these operators would give rise to the following neutral tetron decay modes:

$$
\begin{array}{lc}
\Psi^{0} \rightarrow \tau \tau^{c} h_{d} \phi^{3}, & \Psi^{0} \rightarrow e / \mu e^{c} / \mu^{c} h_{d} \phi^{3}, \\
\Psi^{0} \rightarrow b b^{c} h_{d} \phi^{3}, & \Psi^{0} \rightarrow b b^{c} h_{d} h_{d} h_{u} \phi, \\
\Psi^{0} \rightarrow t t^{c} h_{u} \phi^{3}, & \Psi^{0} \rightarrow t t^{c} h_{u} h_{u} h_{d} \phi, \\
\Psi^{0} \rightarrow c c^{c} d d^{c} \phi^{2}, & \Psi^{0} \rightarrow s s^{c} h_{d} \phi^{3} .
\end{array}
$$

We note that there are several different possible decay modes, any of which may be dominant, depending on unknown features of the model dynamics that determine the relative values of their coefficients. In particular, the most important tetron decays could be into either leptons or quarks, and there are many different possibilities for the dominant flavors.

We plot in Figs. 1-8 below the expected UHECR energy spectra of photons and nucleons due to each of these possible tetron decay modes, as well as the maximum photon fractions expected. The energy spectra were calculated for a mass $M_{X}=2 \times 10^{13} \mathrm{GeV}$, using the fragmentation functions $D^{i}\left(x, M_{X}^{2}\right)$ generated by the code
SHdecay [16]. This code calculates the fragmentation into the seven stable MSSM particles ( $p, \gamma, e$, neutralino LSP $\left.\chi, \nu_{e}, \nu_{\mu}, \nu_{\tau}\right)$ for any given initial decay parton. The many-body decays distribute the total decay energy $M_{X}$ among the different particles. We include Higgs decays, but we ignore the decays of the singlet fields, except to take into account their kinematical effects on the primary quark and lepton spectra. We follow [32] in estimating the probability density $\rho_{n}(z)$ that one decay parton carries off a fraction $z$ of the total available decay energy $M_{X}$ :

$$
\rho_{n}(z)=(n-1)(n-2) z(1-z)^{n-3}
$$

for $n \geq 3$ decay partons. The resulting flux from the emission of a given decay parton is then

$$
E^{3} J^{i}(E)=B x^{3} \int_{x}^{1} \frac{d z}{z} \rho_{n}\left(\frac{x}{z}\right) D^{i}\left(z, M_{x}^{2}\right),
$$

where $i=\left(p, \gamma, e, \chi, \nu_{e}, \nu_{\mu}, \nu_{\tau}\right)$.

To obtain the total UHECR spectrum, we add to this the background flux of nucleons that would be expected to result from a homogenous distribution of extragalactic sources that exhibits the distinctive pileup due to the
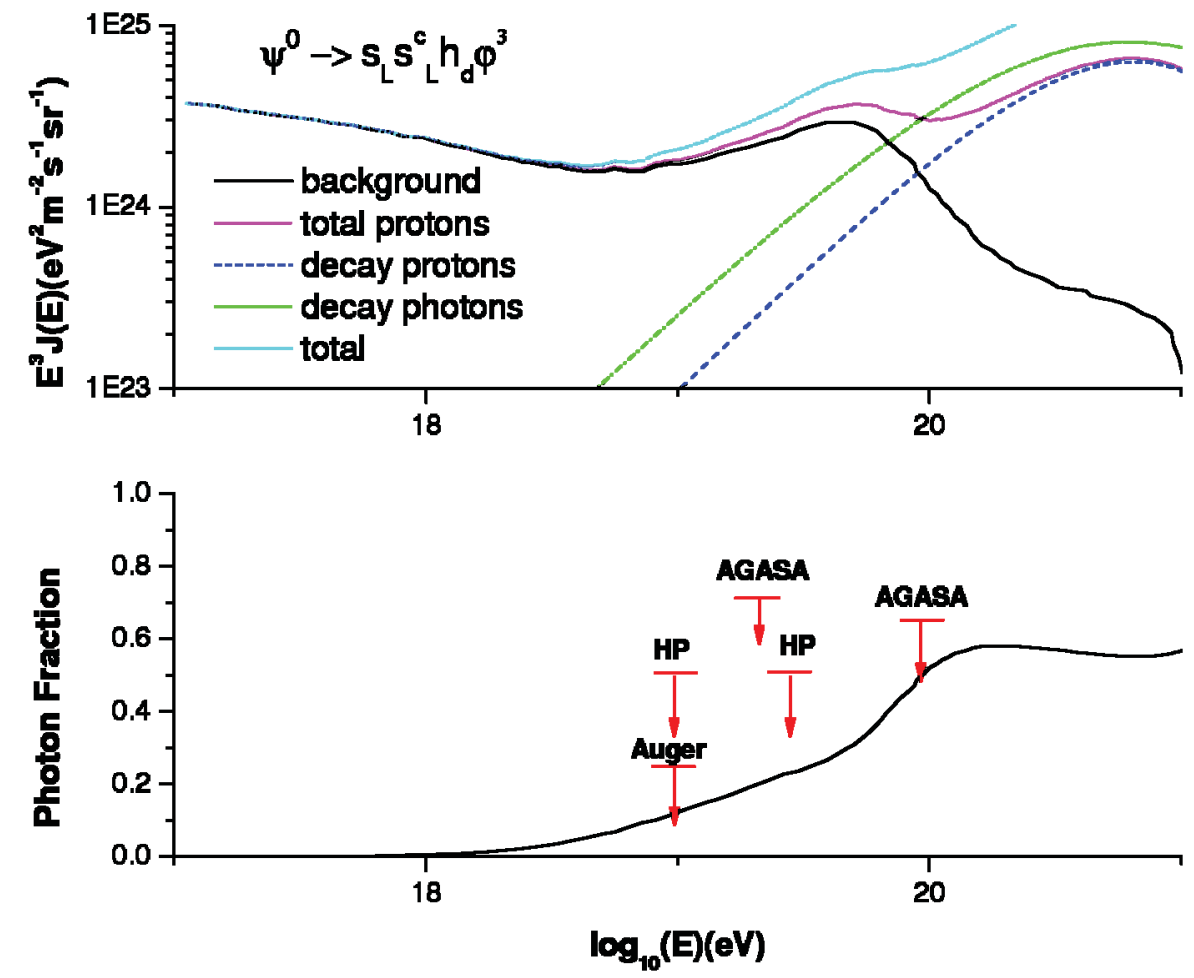

FIG. 1 (color). The top panel shows the total UHECR spectrum and the bottom panel the photon fraction from the decay mode $\Psi^{0} \rightarrow s s^{c} h_{d} \phi^{3}$. 
JOHN ELLIS, V.E. MAYES, AND D. V. NANOPOULOS
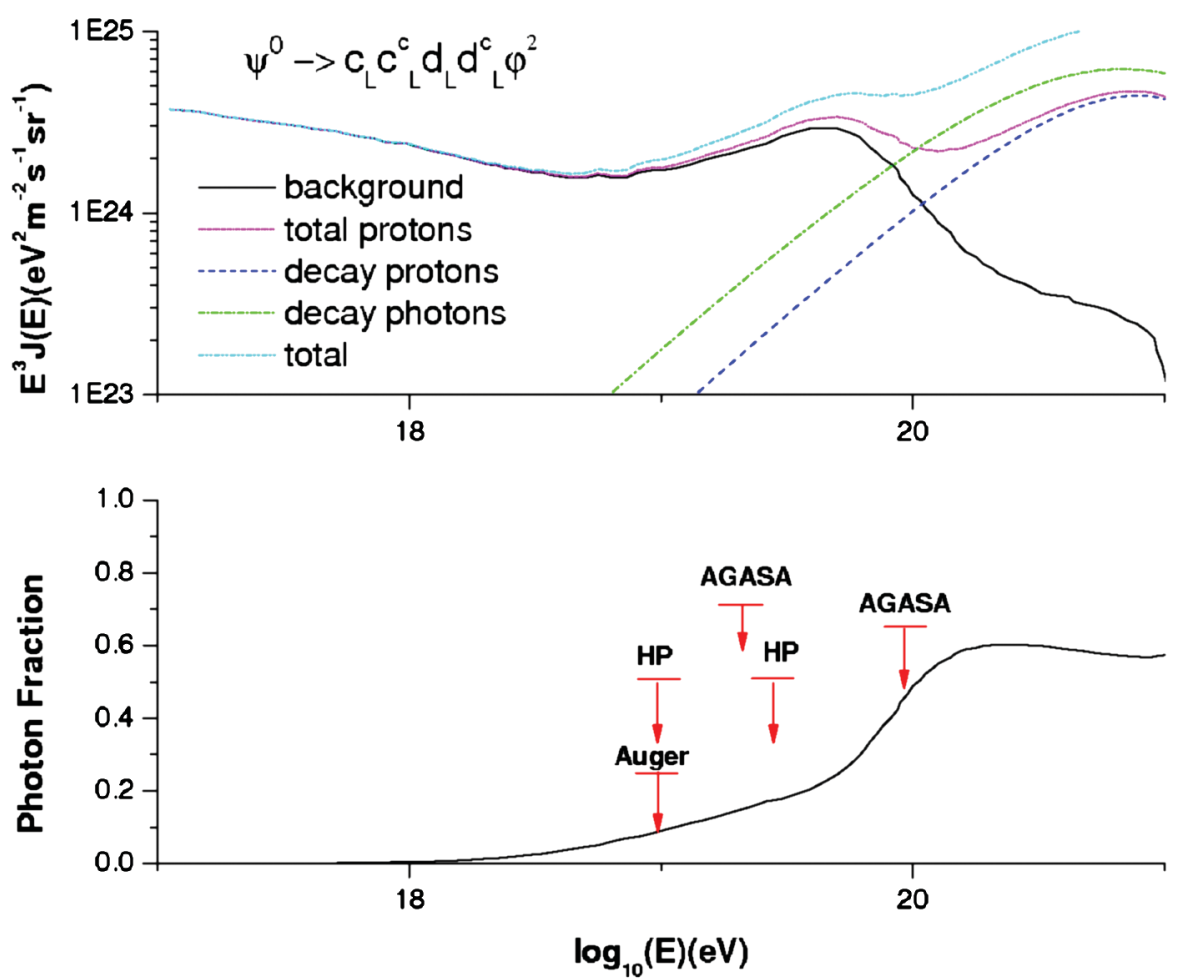

FIG. 2 (color). The top panel shows the total UHECR spectrum and the bottom panel the photon fraction from the decay mode $\Psi^{0} \rightarrow c c^{c} d d^{c} \phi^{2}$.
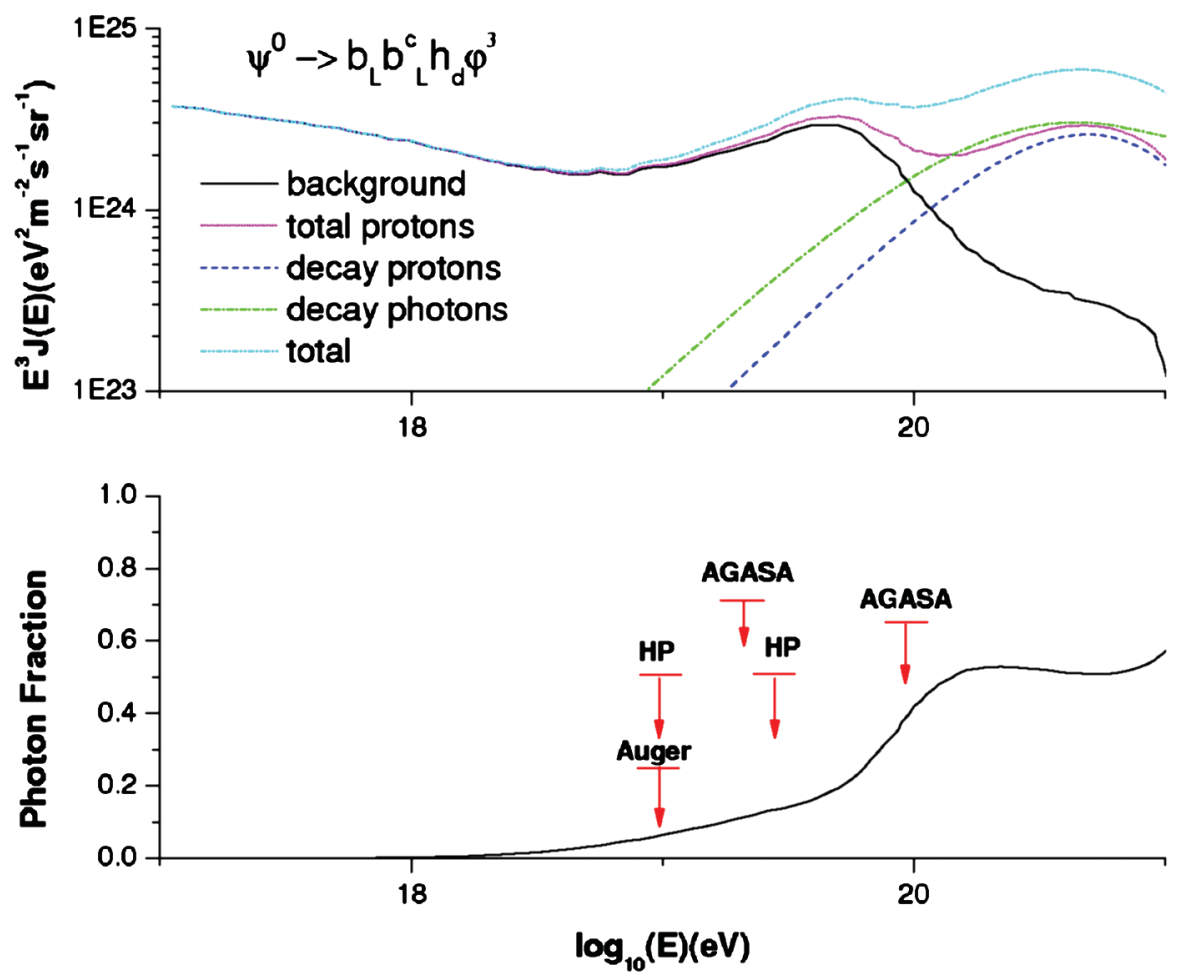

FIG. 3 (color). The top panel shows the total UHECR spectrum and the bottom panel the photon fraction from the decay mode $\Psi^{0} \rightarrow b b^{c} h_{d} \phi^{3}$. 

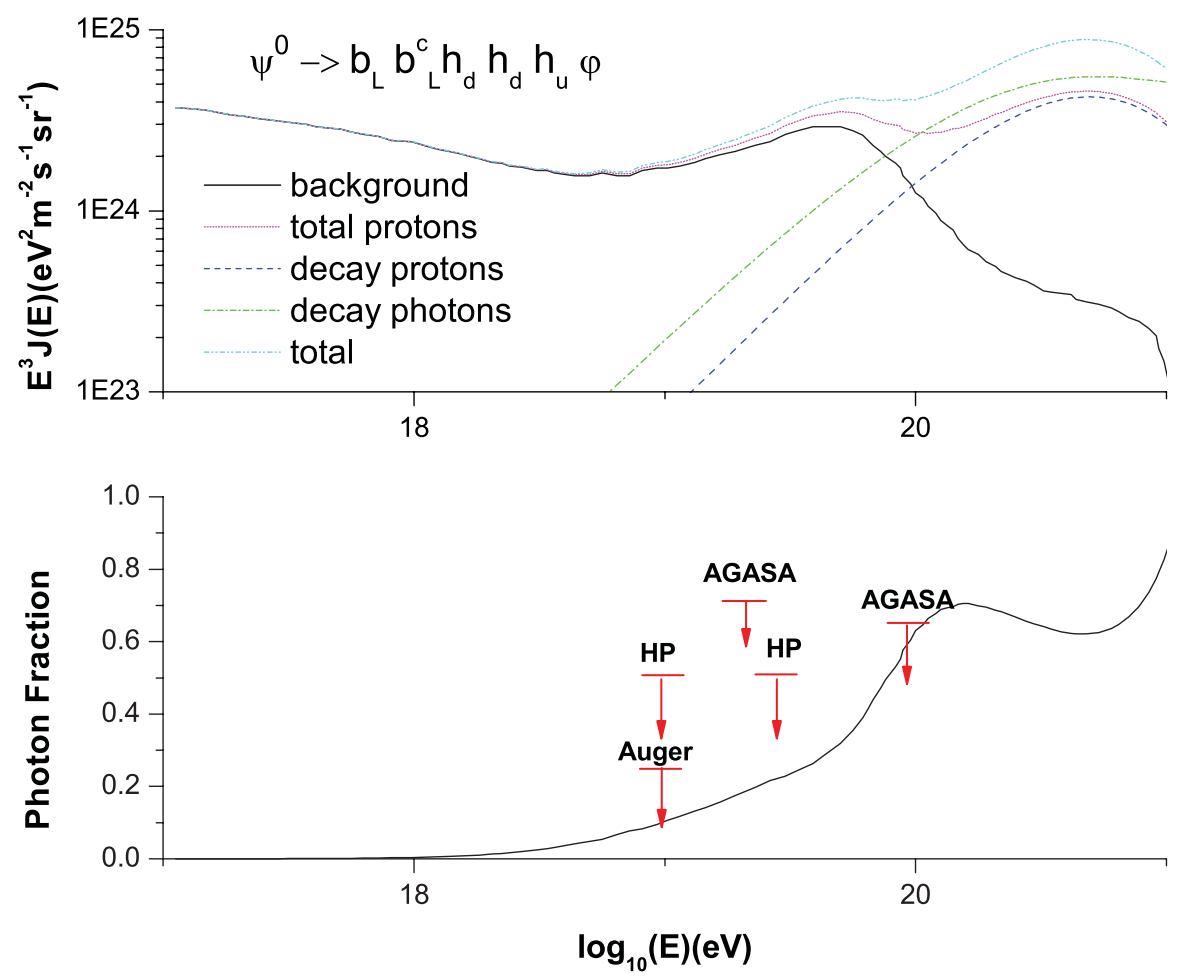

FIG. 4 (color). The top panel shows the total UHECR spectrum and the bottom panel the photon fraction from the decay mode $\Psi^{0} \rightarrow b b^{c} h_{d} h_{d} h_{u} \phi$.
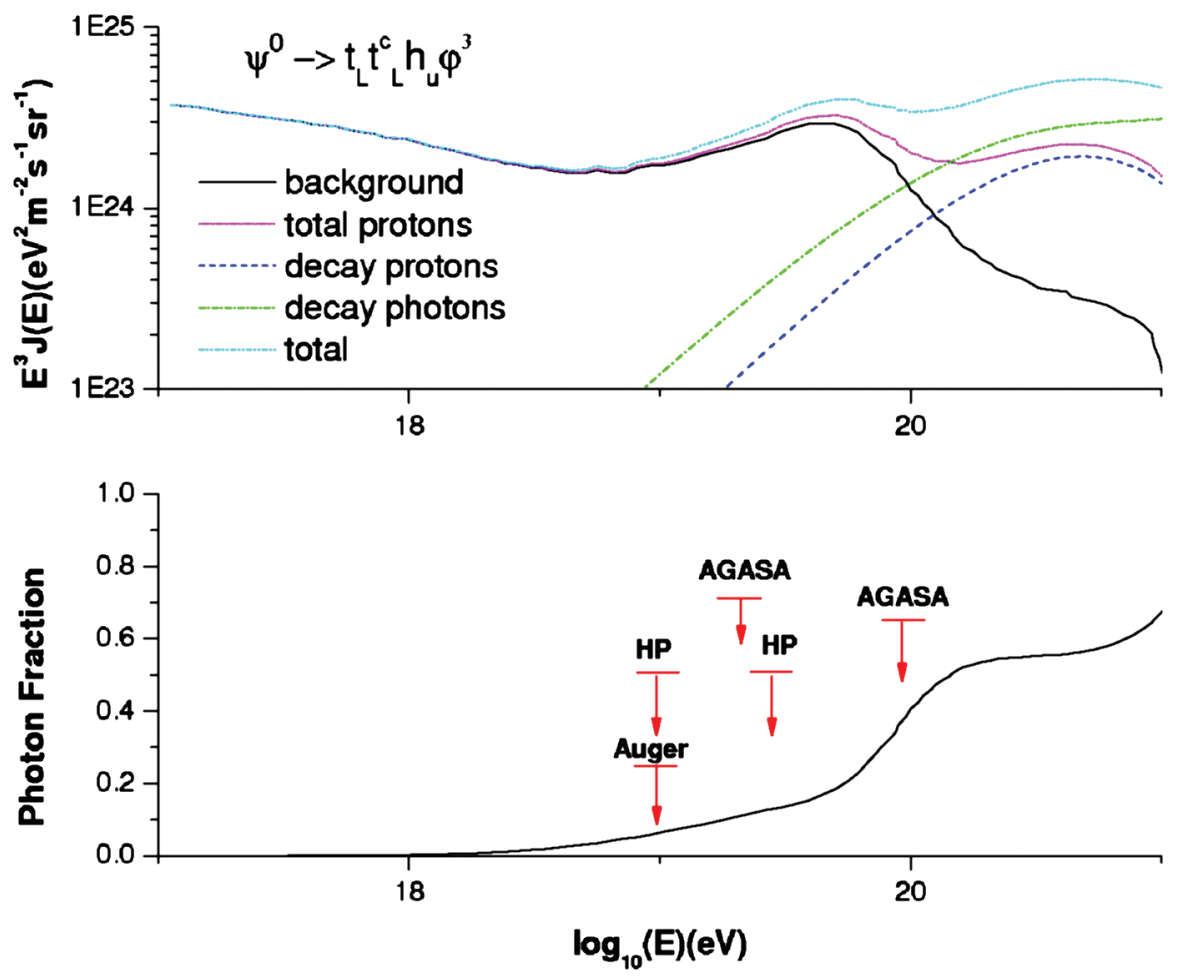

FIG. 5 (color). The top panel shows the total UHECR spectrum and the bottom panel the photon fraction from the decay mode $\Psi^{0} \rightarrow t t^{c} h_{u} \phi^{3}$. 
JOHN ELLIS, V.E. MAYES, AND D. V. NANOPOULOS

PHYSICAL REVIEW D 74, 115003 (2006)
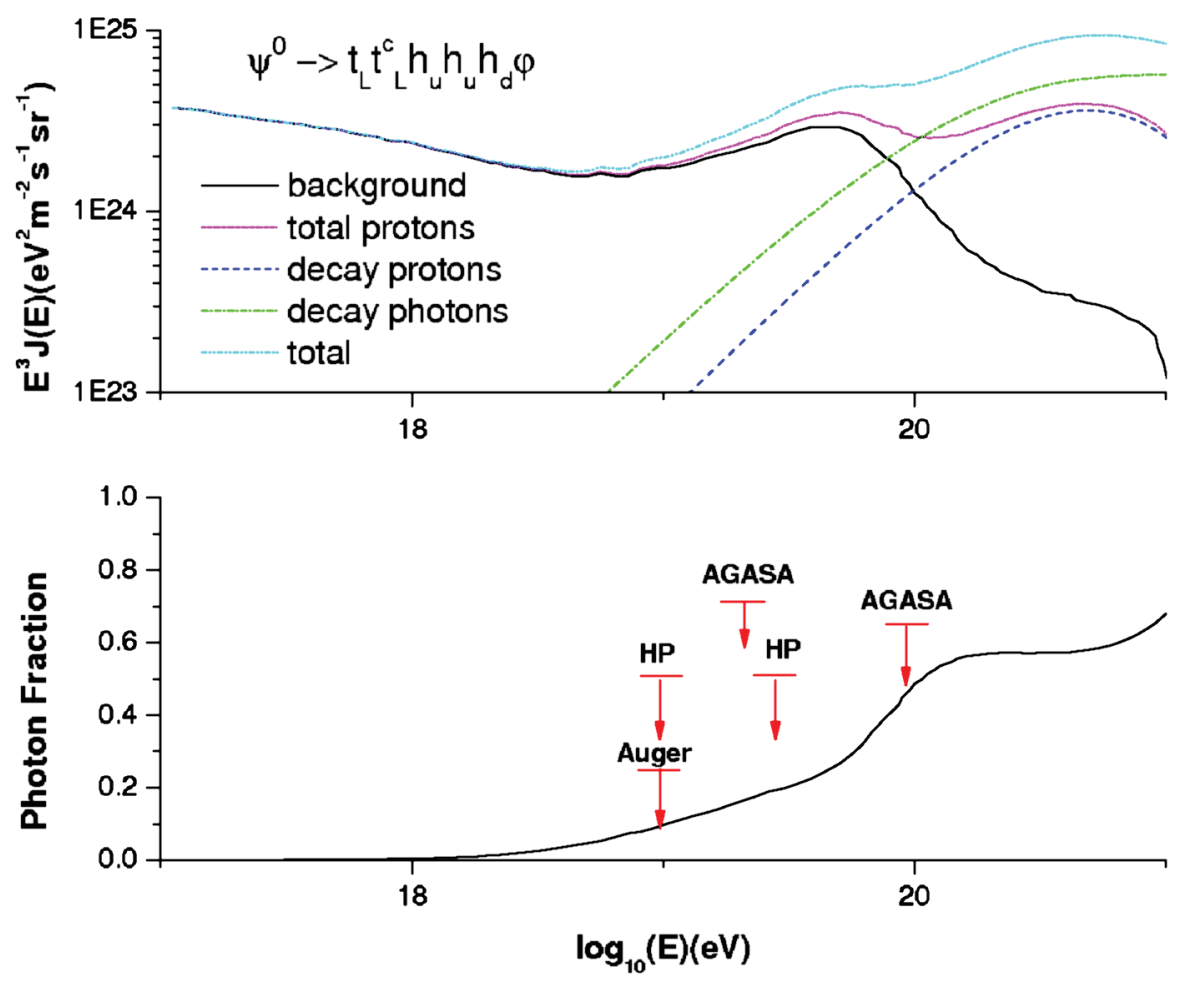

FIG. 6 (color). The top panel shows the total UHECR spectrum and the bottom panel the photon fraction from the decay mode $\Psi^{0} \rightarrow t t^{c} h_{u} h_{u} h_{d} \phi$.
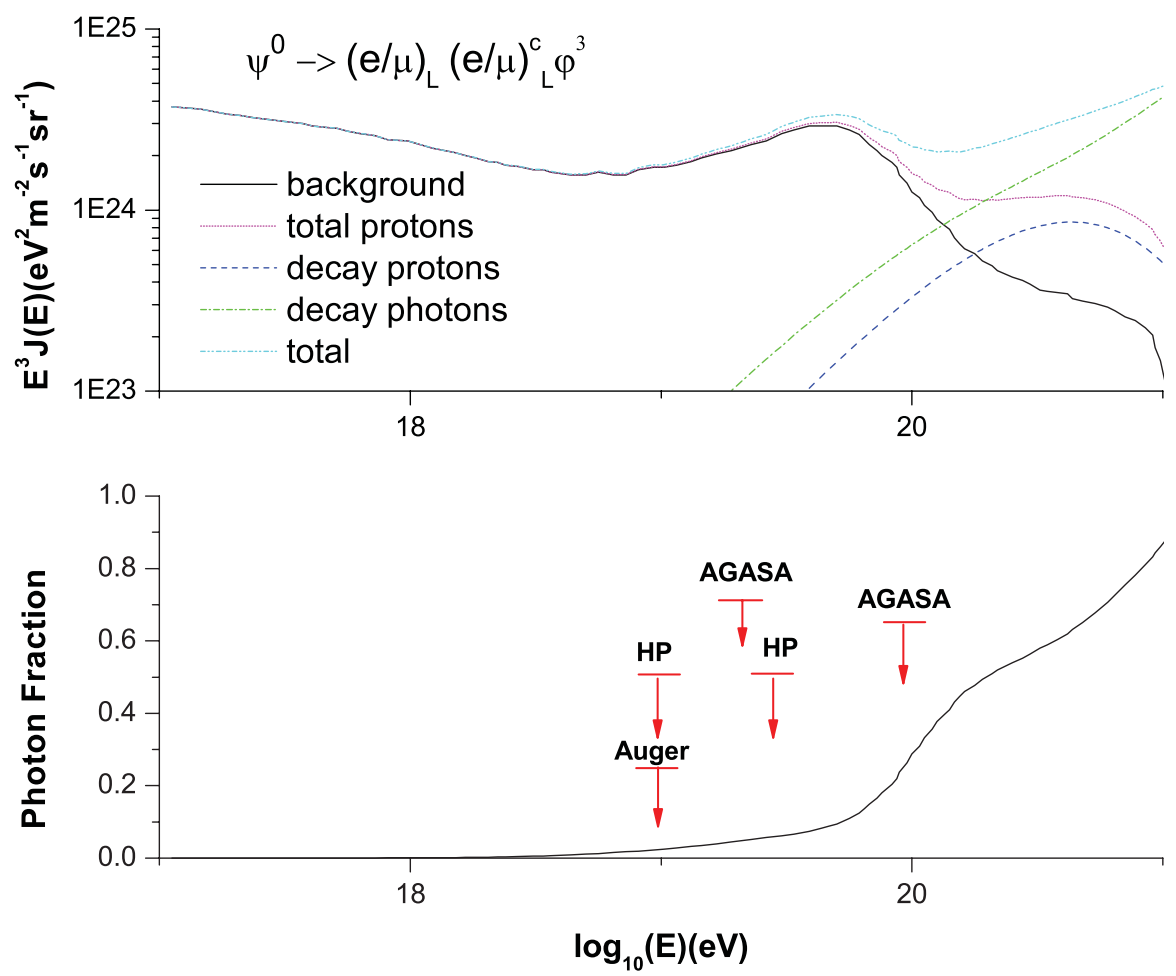

FIG. 7 (color). The top panel shows the total UHECR spectrum and the bottom panel the photon fraction from the decay mode $\Psi^{0} \rightarrow e / \mu e^{c} / \mu^{c} h_{d} \phi^{3}$. 

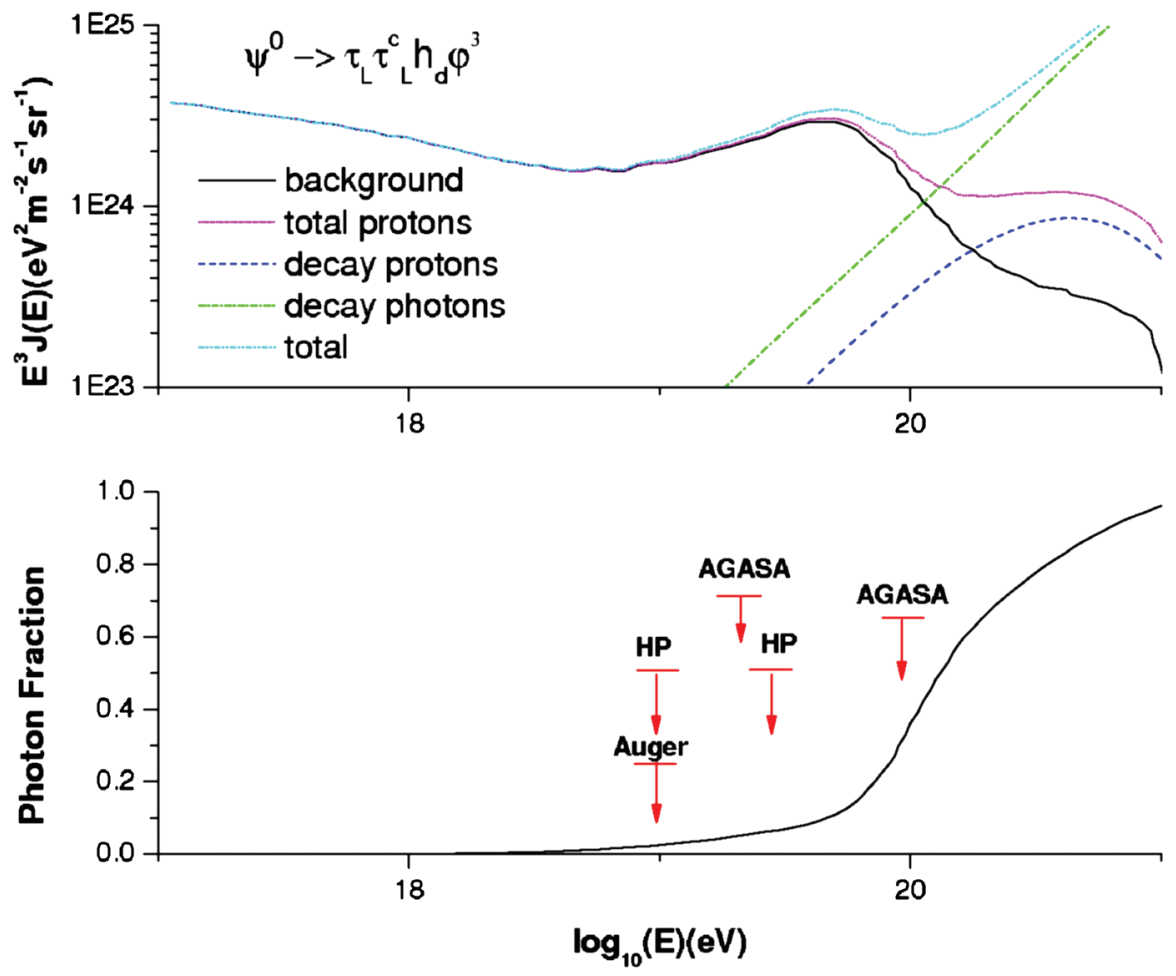

FIG. 8 (color). The top panel shows the total UHECR spectrum and the bottom panel the photon fraction from the decay mode $\Psi^{0} \rightarrow \tau \tau^{c} h_{d} \phi^{3}$.

GZK effect [23]. ${ }^{2}$ The constant $B$ in (19) is a normalization coefficent determined by the tetron number density and lifetime, viz.

$$
B \sim R^{\text {halo }} \frac{n_{X}}{\tau_{X}} \frac{1}{M_{X}} .
$$

This dimensional coefficient $B$ is not determined a priori, and must be fitted to the experimental data. In each of Figs. 1-8, we show the total spectrum obtained by summing the background and the fluxes of nucleons and photons resulting from tetron decay, and in a second panel we display the gamma fractions: $\gamma /(\gamma+p)$. We have assumed no photon attenuation in the calculated spectra, although a strong attenuation cannot be excluded [34], because the galactic radio background has never been accurately measured and its intensity is largely unknown [35].

Figures 1-6 are for quark primaries, and are ordered according to the masses of the quarks involved. In the case of the $b$ quark, we show in Figs. 3 and 4 plots for two superpotential operators with different numbers of accompanying Higgs fields: the two plots are rather similar, and the same is true of the two plots shown in Figs. 5 and 6 for

\footnotetext{
${ }^{2}$ However, we note that this model is likely to come under pressure from upper limits on high-energy cosmic-ray neutrinos [33] - private communication from S. Sarkar, see also http://www-thphys.physics.ox.ac.uk/users/SubirSarkar/ talks/munich05.pdf.
}

primary $t$ quarks. We are thus led to hope that including the (model-dependent) decays of the singlets $\phi$ would not have large effects. The plots for lepton primaries shown in Figs. 7 and 8 are more distinctive, in that the photon fractions rise to much larger values at energies above $10^{20} \mathrm{eV}^{3}$

In Fig. 9 we compare the spectrum for one of the operators with primary $b$ quarks, calculated for a crypton mass of $10^{13} \mathrm{GeV}$, with experimental data from the Fly's Eye, HiRes, AGASA, and Auger experiments [3,5,7,8]. The AGASA flux has been scaled by a factor of 0.55 for consistency with the other data, and the normalizations for the crypton decay contributions to these spectra has been adjusted for the different crypton masses. The limited statistics for UHECRs with energies $\geq 10^{19} \mathrm{eV}$ available in the present data sets do not offer any clear discrimination between crypton masses in the range $2 \times 10^{13} \mathrm{GeV} \geq$ $M_{X} \geq 10^{12} \mathrm{GeV}$. In the case of a crypton mass $\sim 10^{12} \mathrm{GeV}$ there is no clear signal of a crypton contribution to the UHECR since the flux from such a decay is essentially buried within the background from homogenous extragalatic sources. A clear signal of crypton decay, at least in this model, would require a lower limit on the crypton mass $M_{X} \geq 5 \times 10^{12} \mathrm{GeV}$ in order to provide an

\footnotetext{
${ }^{3}$ The photon fractions for second-generation quark primaries look somewhat flatter than those for $b$ and $t$ quarks, but this difference is probably within the modelling uncertainties.
} 


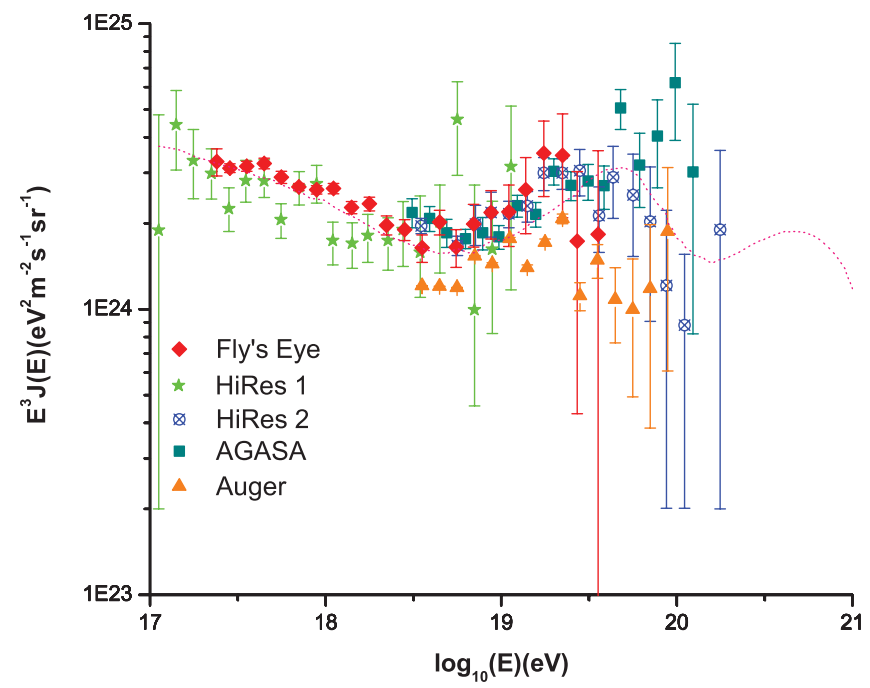

FIG. 9 (color). A comparison with the available data on the UHECRs from the Fly's Eye, HiRes, AGASA, and Auger experiments with the crypton decay model $\Psi^{0} \rightarrow b b^{c} h_{d} \phi^{3}$ for $M_{X}=$ $10^{13} \mathrm{GeV}$.

excess of events above $4 \times 10^{19} \mathrm{eV}$ that could not be attributable to extragalactic astrophysical acceleration mechanisms.

\section{CONCLUSIONS}

We have carried as far as is possible at present the modelling of flipped crypton decay contributions to
UHECRs, including all the possible 10th-order superpotential operators. The experimental data presently available are consistent with all the decay modes possible in this crypton framework. The total UHECR spectra are consistent with a contribution from cryptons weighing between $2 \times 10^{13} \mathrm{GeV}$ and $10^{12} \mathrm{GeV}$, although only a crypton mass $M_{X} \geq 5 \times 10^{12} \mathrm{GeV}$ would provide an unambiguous signal over conventional explanations. The available upper limits on the possible photon fraction do not exclude any of the crypton models we have studied.

In the future, the larger data set expected from Auger may be able to discriminate between crypton decays and other models of UHECRs, and also among different crypton models themselves. Greater statistics will enable the UHECR anisotropy to be measured with sufficient accuracy to discriminate crypton decay from a uniform distribution of astrophysical sources, and more accurate measurements of the photon fraction at higher energies might offer some discrimination between models with lepton and quark primaries, as seen by comparing Figs. 1-6 with Figs. 7 and 8 above.

Thus there is hope that, in the near future, we may finally learn whether UHECRs have a macrophysical origin or a microphysical origin and, in the latter case, may start to discriminate between different microphysical models.

\section{ACKNOWLEDGMENTS}

The work of D.V.N. is supported by DOE Grant No. DE-FG03-95-ER-40917.
[1] K. Greisen, Phys. Rev. Lett. 16, 748 (1966).

[2] G. T. Zatsepin and V. A. Kuzmin, Pis'ma Zh. Eksp. Teor. Fiz. 4, 114 (1966) [JETP Lett. 4, 78 (1966)].

[3] M. Nagano and A. A. Watson, Rev. Mod. Phys. 72, 689 (2000).

[4] For a recent review, see: S. Westerhoff, Int. J. Mod. Phys. A 21, 1950 (2006).

[5] M. Takeda et al., Phys. Rev. Lett. 81, 1163 (1998); Astropart. Phys. 19, 447 (2003).

[6] N. W. Evans, F. Ferrer, and S. Sarkar, Astropart. Phys. 17, 319 (2002).

[7] R.U. Abbasi et al. (High Resolution Fly's Eye Collaboration), Phys. Rev. Lett. 92, 151101 (2004); Phys. Lett. B 619, 271 (2005).

[8] P. Sommers (Pierre Auger Collaboration), astro-ph/ 0507150 .

[9] E. W. Kolb, A. D. Linde, and A. Riotto, Phys. Rev. Lett. 77, 4290 (1996); B. R. Greene, T. Prokopec, and T. G. Roos, Phys. Rev. D 56, 6484 (1997); E. W. Kolb, A. Riotto, and I. I. Tkachev, Phys. Lett. B 423, 348 (1998); D. J.H. Chung, E. W. Kolb, and A. Riotto, Phys. Rev. D 59, 023501 (1998).
[10] A. N. Schellekens, Phys. Lett. B 237, 363 (1990).

[11] J. R. Ellis, J. L. Lopez, and D. V. Nanopoulos, Phys. Lett. B 247, 257 (1990); K. Benakli, J. R. Ellis, and D. V. Nanopoulos, Phys. Rev. D 59, 047301 (1999).

[12] J. R. Ellis, V. E. Mayes, and D. V. Nanopoulos, Phys. Rev. D 70, 075015 (2004).

[13] M. Risse (Pierre Auger Collaboration), astro-ph/0507402.

[14] S. Sarkar (private communication).

[15] M. Risse et al., Phys. Rev. Lett. 95, 171102 (2005).

[16] C. Barbot, hep-ph/0308028.

[17] V. Berezinsky, M. Kachelriess, and A. Vilenkin, Phys. Rev. Lett. 79, 4302 (1997).

[18] V. A. Kuzmin and V. A. Rubakov, Yad. Fiz. 61, 1122 (1998) [Phys. At. Nucl. 61, 1028 (1998)].

[19] M. Birkel and S. Sarkar, Astropart. Phys. 9, 297 (1998).

[20] V. Berezinsky and M. Kachelriess, Phys. Rev. D 63, 034007 (2001).

[21] C. Barbot and M. Drees, Phys. Lett. B 533, 107 (2002).

[22] R. Aloisio, V. Berezinsky, and M. Kachelriess, Phys. Rev. D 74, 023516 (2006).

[23] V. Berezinsky, A.Z. Gazizov, and S. I. Grigorieva, Phys. Lett. B 612, 147 (2005). 
[24] H. Georgi and S. L. Glashow, Phys. Rev. Lett. 32, 438 (1974).

[25] S. M. Barr, Phys. Lett. B 112, 219 (1982).

[26] J.P. Derendinger, J. E. Kim, and D. V. Nanopoulos, Phys. Lett. B 139, 170 (1984).

[27] I. Antoniadis, J.R. Ellis, J.S. Hagelin, and D. V. Nanopoulos, Phys. Lett. B 231, 65 (1989); 208, 209 (1988); 213, 562 (1988); 205, 459 (1988); 194, 231 (1987); 205, 459 (1988)

[28] J. R. Ellis, D. V. Nanopoulos, and J. Walker, Phys. Lett. B 550, 99 (2002).

[29] K. S. Narain, Phys. Lett. B 169, 41 (1986); K. S. Narain, M. H. Sarmadi, and E. Witten, Nucl. Phys. B279, 369
(1987).

[30] J.L. Lopez and D. V. Nanopoulos, Phys. Rev. Lett. 76, 1566 (1996).

[31] C. Coriano, A. E. Faraggi, and M. Plumacher, Nucl. Phys. B614, 233 (2001).

[32] S. Sarkar and R. Toldra, Nucl. Phys. B621, 495 (2002).

[33] M. Ahlers, L. A. Anchordoqui, H. Goldberg, F. Halzen, A. Ringwald, and T. J. Weiler, Phys. Rev. D 72, 023001 (2005).

[34] S. Sarkar, Acta Phys. Pol. B 35, 351 (2004).

[35] R. J. Protheroe and P. L. Biermann, Astropart. Phys. 6, 45 (1996); 7, 181(E) (1997). 